\title{
Comparison between multi-azimuth, wide-azimuth, and full-azimuth towed-streamer acquisition: a full 3D finite-difference study in the Santos Basin
}

Franck Le Diagon*, Nick Moldoveanu, Eugene Gridnev, Jeff Thompson, and Luis Arechiga Salinas, WesternGeco

Copyright 2017, Society of Exploration Geophysicists. This paper was presented at the 2017 SEG International Exposition and 87th Annual Meeting and is presented by permission of SEG in the 16th International Congress of the Brazilian Geophysical Society held in Rio de Janeiro, Brazil, 19-22 August 2019.

Contents of this paper were reviewed by the Technical Committee of the 16th International Congress of the Brazilian Geophysical Society and do not necessarily represent any position of the SBGf, its officers or members. Electronic reproduction or storage of any part of this paper for commercial purposes without the written consent storage of any part of this paper for commercial purposes. without the witten consent prohibited.

\begin{abstract}
A seismic modeling study was performed to analyze and compare the illumination and images generated by different acquisition scenarios in a selected area of the pre-salt province of the Santos Basin, offshore Brazil. Using the available data, a realistic earth model was created to carry out $3 \mathrm{D}$ ray tracing as well as to generate and migrate finite-difference synthetic shot records. The relative merits of several acquisition designs including narrow-, wide-, and full-azimuth acquisition geometries were considered in this modeling study. This paper describes the modeling experiments and discusses the results.
\end{abstract}

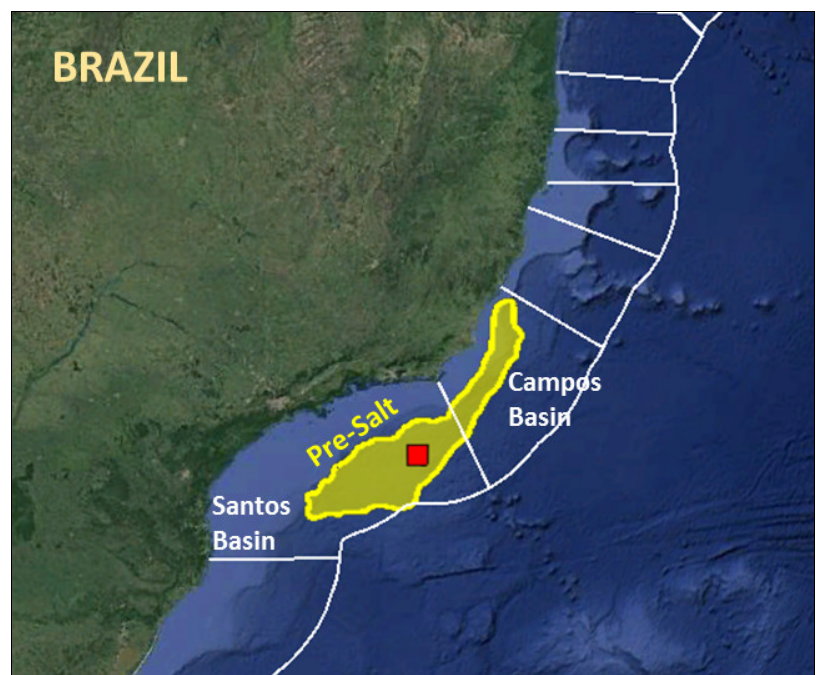

Figure 1: Extent of the pre-salt area (yellow), offshore Brazil. Area selected for modeling study (red).

\section{Introduction}

The pre-salt province covers a large portion of the Santos and Campos Basins (Figure 1) and accounts for more than half of the oil production in Brazil. The pre-salt reservoirs lay below a deep water column and a thick section of evaporates (Figure 2).

Most of the Santos Basin pre-salt fields are covered by 3D seismic exploration surveys. With the exception of a limited number of ocean-bottom surveys and a fullazimuth towed-streamer survey implemented over the lara field in 2010, most of the surveys were acquired by narrow-azimuth geometries, i.e., a single seismic vessel towing two sources and eight to twelve streamers along straight sail lines.

The complexity and thickness of the salt layers constitute a challenge to proper imaging of the pre-salt reservoirs using conventional seismic techniques. This modeling study was carried out to analyze different acquisition geometries and investigate how these geometries could better address these challenges.

\section{Earth model building for modeling}

To simulate the propagation of seismic waves using the two-way wave equation implemented in finite differences, both depth interval velocity and density fields are required. The model was built within an area of approximately $800 \mathrm{~km} 2$ located in a central portion of the pre-salt province (Figure 1). The selected area is covered by a high-definition narrow-azimuth data set acquired in 2013 and by a unique full-azimuth data set acquired in 2011 (Le Diagon et al., 2011).

The available seismic volume (Figure 2a) was used to interpret the top-salt, base-salt, a set of supra-salt, intrasalt, and pre-salt horizons, as well as a set of faults. The interpreted surfaces were then assembled to produce a realistic structural model (Figure $2 b$ ). By implementing an advanced model building workflow recently developed for modeling purposes (Le Diagon et al., 2016), the seismic amplitudes extracted from the available migrated volume were translated into reflectivity, transformed into acoustic impedance, and converted to density values, which were then extrapolated into the structural model to create the density model (Figure 2d). In addition, the structural model was populated with available seismic velocity values to create the velocity model (Figure $2 \mathrm{c}$ ).

Modeling experiments were performed in an iterative process to update or validate the model. At each iteration, $2 \mathrm{D}$ synthetic gathers were generated and migrated to 
produce a $2 \mathrm{D}$ migrated section (Figure $3 \mathrm{c}$ ), which was then compared to a section extracted from the available $3 \mathrm{D}$ migrated volume (Figure $3 \mathrm{a}$ ) to evaluate the structural and amplitude consistency between real and synthetic data.

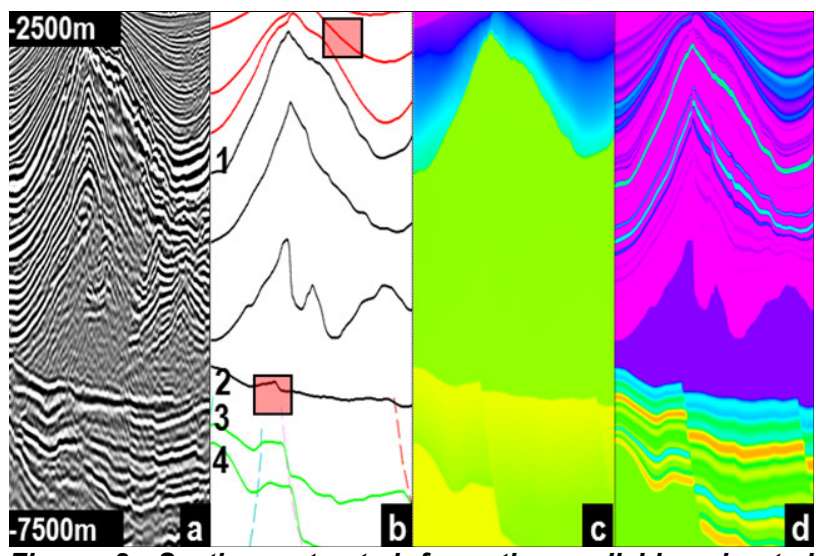
Figure 2: Section extracted from the available migrated volume (a); from the derived structural model (b); from the derived velocity model (c); from the derived density model (d). Interpreted horizons, top salt (b1), bottom salt (b2), top syn-rift (b3), and bottom syn-rift (b4).

The analysis of the synthetic experiments also revealed that the seismic events observed within the halite section, colored in red on Figure 3, represent, internal multiple energy generated within the overlying evaporate section. Further analyses also indicated that these multiples considerably affect the image of the pre-salt section.

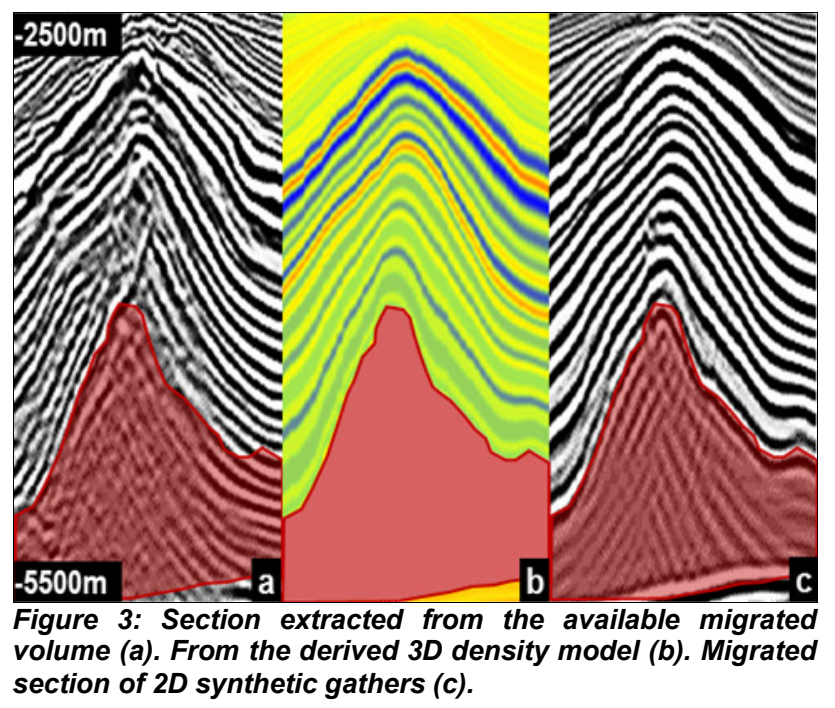

\section{Design of acquisition geometries}

Various acquisition scenarios were considered for analysis and modeling. Herein, we will carefully look at four of these acquisition geometries (Table 1):
- G1 represents a typical example of an old legacy narrow-azimuth (NAZ) acquisition geometry limited to eight cables and a 6000-m offset. The shooting direction is in the strike direction (Figure 4a).

- G2 represents the same acquisition geometry as G1 shooting in the dip direction (Figure 4b).

- G3 represents a four-vessel wide-azimuth (WAZ) geometry commonly deployed in subsalt areas such as the Gulf of Mexico, oriented perpendicular to the legacy geometry (Figure 4c).

- G4 represents a dual-vessel circular shooting geometry where each vessel sails along 6500-m radius circles separated by $1200 \mathrm{~m}$ (Figure 4d). Each set of circle centers is positioned on a grid of $1700 \times 1700 \mathrm{~m}$. The main vessel, towing both sources and streamers, is positioned around $6000 \mathrm{~m}$ behind the source vessel to record long-offset ranges in addition to the short- and mid-offset ranges.

Table 1: Acquisition parameters for each geometry (G1 to G4) illustrated in Figure 4.

\begin{tabular}{|l|l|l|l|l|}
\hline Parameters & G1 & G2 & G3 & G4 \\
\hline Geometry & NAZ & NAZ & WAZ & CIRCULAR \\
\hline Azimuth $\left(^{\circ}\right)$ & 33 & 303 & 303 & Far azimuth \\
\hline Number of vessels & 1 & 1 & 4 & 2 \\
\hline Number of cable vessels & 1 & 1 & 2 & 1 \\
\hline Number of cables & 8 & 8 & 12 & 12 \\
\hline Cable length (m) & 6000 & 6000 & 9000 & 8000 \\
\hline Cable interval (m) & 100 & 100 & 100 & 100 \\
\hline Number of sources & 2 & 2 & 4 & 4 \\
\hline Shot interval flip-flop (m) & 25 & 25 & 25 & 18.75 \\
\hline Cable depth $(\mathbf{m})$ & 10 & 10 & 10 & 10 \\
\hline Source depth $(\mathbf{m})$ & 10 & 10 & 10 & 10 \\
\hline $\begin{array}{l}\text { Sail line interval }(\mathbf{m}) \\
\text { Coil center distance }(\mathbf{m})\end{array}$ & 400 & 400 & 600 & $1700 / 1200$ \\
\hline
\end{tabular}

For consistency purposes, all four acquisition geometries were assigned the same source and receiver depths of 10 $\mathrm{m}$ and the same flip-flop shot interval of $25 \mathrm{~m}$, except the circular geometry (G4), which was assigned a flip-flop shot interval of $18.75 \mathrm{~m}$. The distance between vessels and the deep water bottom allows fitting one far-offset record between two near-offset shots separated by an interval of $2 \times 18.75 \mathrm{~m}$ by adjusting the far-offset shot delay and by overlapping records.

The nominal source and receiver positions were calculated and plotted, taking into account a target or image area of $100 \mathrm{~km} 2$ and a migration fringe of $4500 \mathrm{~m}$ around the target area. A set of preplots was generated for each of the acquisition geometries. 


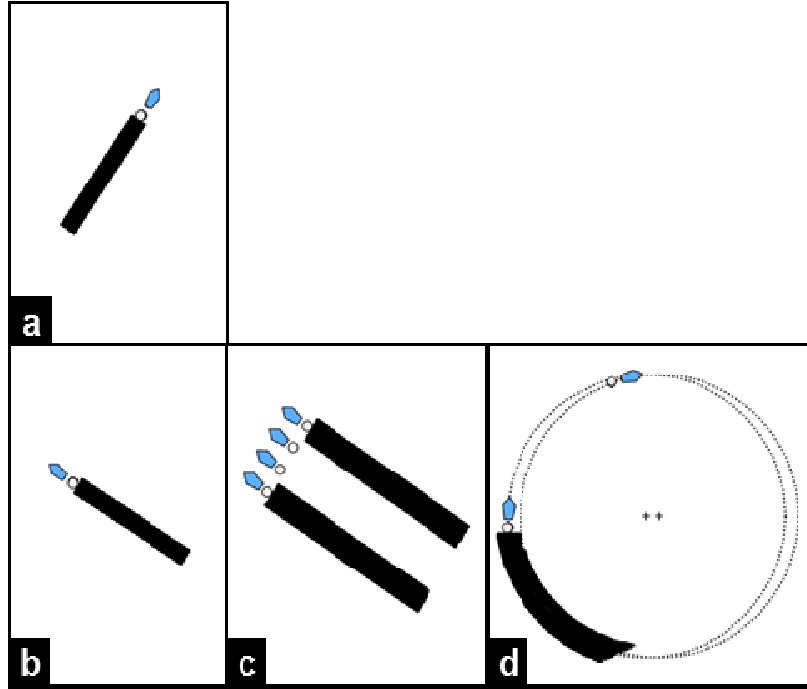

Figure 4: Seismic acquisition geometries; (a) narrowazimuth geometry as described in Table 1, column G1; (b) narrow-azimuth geometry as described in Table 1, column G2; (c) wide-azimuth geometry as described in Table 1, column G3; (d) full-azimuth geometry as described in Table 1 , column G4, 6500-m circle radius.

\section{Description of the modeling experiments}

A ray-tracing study was performed to evaluate the illumination produced by each acquisition geometry on the target surface. The top syn-rift was selected as the target surface (Figure 2b). In this experiment, the rays were propagated from each shot location through the velocity model, reflected at the target surface, and propagated back to the receiver locations. The hit count and illumination amplitude values were calculated by the ray-tracing algorithm and mapped onto the target surface (Figures $5 \mathrm{a}$ to $5 \mathrm{~d}$ ). In addition, the illumination maps produced by the last three geometries G2, G3, and G4 were individually summed to the illumination map produced by the first geometry G1 to simulate the illumination uplift achieved by merging two surveys (Figures 5 e to $5 \mathrm{~g}$ ).

Table 2: Parameters implemented for the modeling and migration experiments.

\begin{tabular}{|l|l|}
\hline Parameters & \\
\hline Record length (ms) & 9000 \\
\hline Maximum depth (m) & 8500 \\
\hline Aperture (m) & 4500 \\
\hline Maximum frequency (Hz) & 30 \\
\hline Surface multiples & yes \\
\hline Source and receiver ghosts & yes \\
\hline
\end{tabular}

In a second stage, a wave propagation modeling study was undertaken to evaluate the image produced by each acquisition geometry. The modeling parameters were estimated by analyzing real data and running a set of synthetic tests. These parameters are listed in Table 2. For each set of preplots, a full set of synthetic gathers were generated by implementing a finite-difference modeling code, and subsequently migrated in depth by applying a reverse time migration algorithm. Sections extracted from the migrated volumes within a deep window are shown in Figure 6.

In addition, the migrated images produced by the last three geometries $\mathrm{G} 2, \mathrm{G} 3$, and $\mathrm{G} 4$ were summed to the migrated image produced by the first geometry $\mathrm{G} 1$ to simulate the imaging uplift achieved by merging two surveys. Figure 7 presents these results within a shallow window.

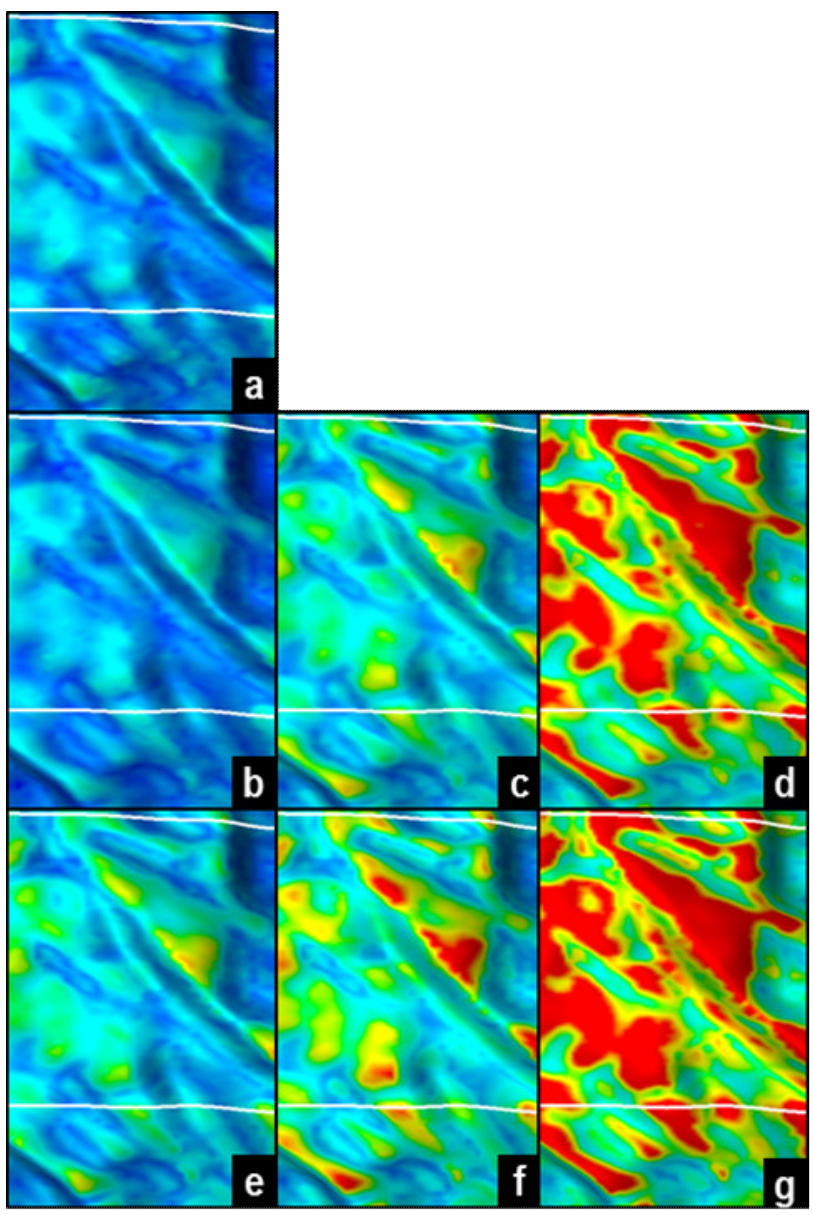

Figure 5: Illumination amplitudes mapped on the target surface produced by geometry G1 (a), geometry G2 (b), geometry G3 (c), geometry G4 (d), geometries G1 plus G2 (e), geometries G1 plus G3 (f), geometries G1 plus G4 (g). The white lines represent the north and south edges of the target area.

\section{Modeling results}

The analysis of the illumination and modeling results allowed us to arrive at the following conclusions and recommendations.

- As expected, the target surface illumination was improved by making the acquisition design longer and wider, and further improved by making it full azimuth (Figure $5 a$ to $d$ ). 
- The circular geometry G4 produced a better target surface illumination due to the full-azimuth coverage, longer offsets and high density of shots and receivers (Figure 5d).

- The narrow-azimuth geometry G1 produced a relatively weak target surface illumination (Figure 5a). By adding an orthogonal pass, the illumination (Figure $5 \mathrm{e}$ ) is improved to a level almost comparable to the illumination produced by the wide-azimuth geometry $\mathrm{G} 3$ (Figure 5c).

- In the deeper section, the quality of the synthetic image produced by the narrow-azimuth geometries G1 and G2 is poor due to the noise content (Figures $6 a$ and $6 b)$. The images produced by the wide- and full-azimuth geometries G3 and G4, respectively, revealed a better continuity of the events and a superior fault delineation (Figures 6c to 6d).

- In the shallower section, the image produced by the wide-azimuth geometry G3 is relatively noisier compared to the other images (Figures $7 \mathrm{~b}$ to $7 \mathrm{~d}$ ). The quality of the image is improved by summing the first migrated volume (Figures $7 \mathrm{e}$ to $7 \mathrm{~g}$ ).

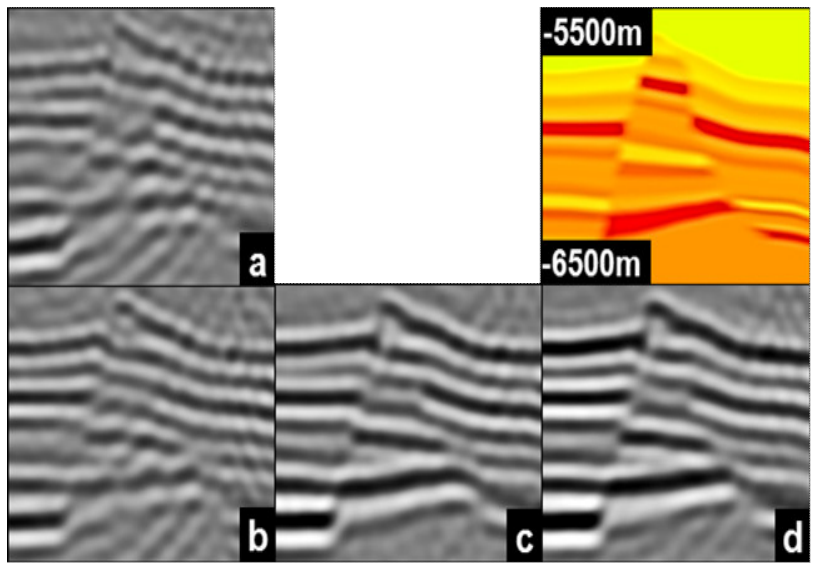

Figure 6: Section extracted from the density model (top right) within the deep window indicated by the red box on Figure 1. Section extracted from the migrated volume generated though this model by geometry G1 (a), geometry G2 (b), geometry G3 (c), geometry G4 (d).

\section{Conclusions}

We discussed a seismic modeling study performed in a selected area of the pre-salt province of the Santos Basin. The comparison of different acquisition geometries was based on ray-tracing illumination of a target horizon and on full 3D finite-difference wave-equation acoustic modeling and reverse time migration imaging. The modeling results indicate that implementing a wide- or full-azimuth acquisition geometry in this area is recommended to improve the quality of the pre-salt image. We suggest conducting a further study to investigate dual- and triple-vessel-type geometries that could be used to acquire very efficiently richer-azimuth seismic surveys.

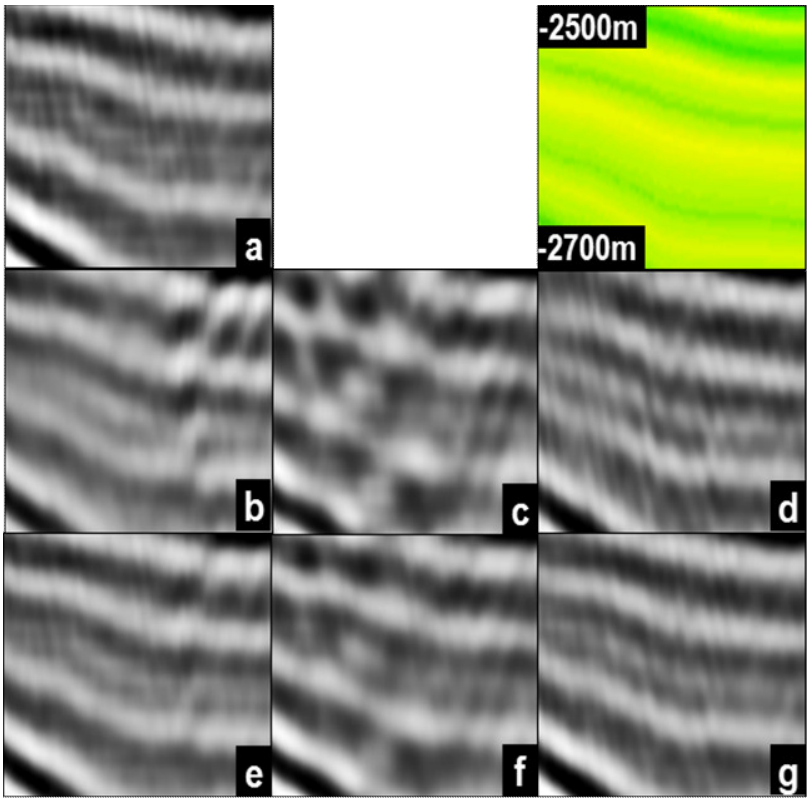

Figure 7: Section extracted from the density model (top right) within the shallow window indicated by the red box on Figure 1. Section extracted from the migrated volume generated though this model by geometry $G 1$ (a), geometry G2 (b), geometry G3 (c), geometry G4 (d), geometries G1 plus G2 (e), geometries G1 plus G3 (f), geometries G1 plus G4 (g).

\section{Acknowledgments}

The authors thank WesternGeco for permission to publish this paper.

\section{References}

Le Diagon, F., J. Soldo, E. C. Mundim, C. E. Lemos, D. C. Garcia, A. Z. N. De Barros, N. Moldoveanu, A. Fenwick, T. Bunting, A. Cooke, and R. D. M. Centeno, 2011, First full-azimuth towed-streamer survey offshore Brazil - an acquisition and survey design case study: Presented at the 12th International Congress of the Brazilian Geophysical Society.

Le Diagon, F., L. A. Salinas, E. S. Casillas, and F. M. Alcántara, 2016, Advanced methods to build complex earth models for seismic modeling: Presented at the International Conference and Exhibition, AAPG. 